\title{
Características de la asistencia psicológica en el contexto universitario: la Clínica Universitaria de la UAM
}

\author{
Ana Calero Elvira, Marta de la Torre Martí, Rebeca Pardo Cebrián \\ Universidad Autónoma de Madrid \\ Marta Santacreu Ivars \\ Universidad Europea de Madrid
}

RESUMEN

En el presente estudio se describe la tipología, las características sociodemográficas de los clientes y los tratamientos psicológicos aplicados en la Clínica Universitaria del CPA-UAM. La muestra está formada por 309 clientes, de los cuáles 255 finalizan el tratamiento. La mayoría de los clientes obtienen alta terapéutica $(65,1 \%)$, con una duración de 17 sesiones de media. Los problemas clínicos más frecuentes son ansiedad $(36,8 \%)$ y bajo estado de ánimo (13,3\%). El tratamiento, realizado por psicólogos inexpertos bajo supervisión, logra una mejoría media significativa del nivel de funcionamiento de los clientes (28,26 puntos más en el EEAG, escala de 0-100) y una valoración media muy alta del éxito en el tratamiento por los clientes y los psicólogos (9,49 y 9,02 escala de 0-10, respectivamente). En conjunto, se discuten las implicaciones relativas a la práctica y la investigación clínica, y se destaca el papel de las clínicas universitarias como nexo entre ambos campos de la Psicología.

Palabras clave: clínica universitaria; tratamientos psicológicos; terapia cognitivo-conductual; características del tratamiento.

\section{ABSTRACT}

The current study describe the typology, sociodemographic characteristics and psychological treatments applied in the CPA-UAM University Clinic. The sample is made of 309 cases, of which 255 completed the treatment. The majority of clients who started psychological treatment (65.1\%) received the therapeutic release, after receiving 17 sessions on average. The most frequent clinical problems are anxiety (36.8\%) and low mood (13.3\%). Treatments are applied by inexperienced psychologists under supervision. On average, there is significant improvement in the functioning level of clients (28.26 points more in the EEAG, scale of 0-100) and the treatment is evaluated as very satisfactory by clients and psychologists (9.49 and 9.02, scale of 0-10, respectively). Finally, the implications for practice and clinical research are discussed, and the role of university clinics as a link between both fields of psychology is highlighted.

Keywords: University clinic; psychological treatments; cognitive-behavior therapy; treatment characteristics. 


\section{Introducción}

El ejercicio de la psicología clínica es entendido como una actividad científico-profesional (APA, 1992; COP, 1993); sin embargo, la disociación entre la investigación y la práctica es un clásico dilema en Psicología (Becoña, 1999; Kazdin, 2008; Westen, 2007). En los últimos veinte años las investigaciones sobre terapias psicológicas empíricamente validadas han experimentado grandes avances, pero la posesión de un conocimiento más exhaustivo no ha supuesto siempre una integración del desarrollo científico en el ámbito aplicado ni ha conllevado progresos terapéuticos claros (Echeburúa, Corral, \& Salaberría, 2010). Indudablemente existen discrepancias entre el ámbito de la investigación y la práctica psicológica pues los objetivos y prioridades de cada uno son diferentes. Las investigaciones sobre eficacia y efectividad buscan encontrar la superioridad de un tratamiento concreto para un tipo de problema y se centran en demostrar la validez de las hipótesis conceptuales que plantean. Por otro lado, la actividad terapéutica busca la forma más efectiva y eficiente de tratar los problemas de la persona que acude a consulta, de acuerdo a una perspectiva ideográfica, con una metodología que se ajuste al paciente, centrada en el pragmatismo (Pérez, Fernández-Hermida, Fernández, \& Amigo, 2003).

En la actualidad, existe un importante debate en torno a si los resultados encontrados sobre la eficacia de los tratamientos psicológicos llevados a cabo en investigaciones controladas podrían generalizarse a la práctica clínica real. Estas investigaciones tienen problemas de validez ecológica, en tanto que las condi- ciones no son representativas del contexto clínico real al no tener en cuenta numerosos aspectos de la práctica clínica (Bower, 2003; Chambless \& Ollendick, 2001). En estos estudios la aplicación del tratamiento se realiza normalmente de forma estandarizada y protocolizada. Estas condiciones se alejan del contexto clínico aplicado en el que las intervenciones se ajustan momento a momento al cliente, aunque los objetivos terapéuticos cambien o aparezcan nuevas demandas en el trascurso del proceso terapéutico. Adicionalmente, en la práctica clínica suele haber mayor comorbilidad psicológica de la prevista en las guías (Bados, García, \& Fusté, 2002; Shean, 2012). Como ya han señalado numerosos autores, también es fundamental estudiar esos resultados en la práctica clínica en contextos naturales (Bados, García, \& Fusté, 2002; Bower, 2003; Chambless, \& Ollendick, 2001; Shean, 2012) aunque estas investigaciones son escasas y tampoco están exentas de obstáculos. Principalmente tienen problemas de validez interna, pues las muestras son sesgadas y heterogéneas, no suele haber grupos control y la evaluación y diagnóstico no se ajustan muchas veces a las exigencias de la investigación.

Dadas las diferencias en estos dos ámbitos de aplicación, se hace necesario conocer las características de la población atendida en la práctica clínica psicológica. Por una parte, posibilitaría el avance y la adaptación de los procedimientos terapéuticos existentes a las demandas de la población y permitiría comparar los resultados en terapia psicológica con los estudios de investigación controlados. En España existen diversos estudios epidemio- 
lógicos sobre los problemas psicológicos que ayudan a contextualizar en nuestro entorno las características sociodemográficas y clínicas de la población. En un estudio de 2006 realizado a 5.473 personas por ESEMeD-España, se mostraba que un $19,5 \%$ de las personas presentaron un problema psicológico en algún momento de su vida y un $8,4 \%$ lo presentó en el último año. Hallaron que el problema psicológico más frecuente era el episodio depresivo mayor, seguido de la fobia específica, el trastorno por abuso de alcohol y la distimia (Haro et al., 2006). En un estudio más reciente (Bones, Pérez, Rodríguez-Sanz, Borrell, \& Obiols, 2010) que analiza los datos de la Encuesta Nacional de Salud en España, realizada a 29.476 personas, se puso de manifiesto que la prevalencia de problemas psicológicos era mayor en mujeres que en hombres y que algunas de las variables asociadas a una mayor prevalencia de problemas eran: estar separado o divorciado, ser inmigrante de un país en vías de desarrollo, tener peores condiciones socioeconómicas, tener poco apoyo social, estar desempleado o en baja laboral y tener enfermedades crónicas.

En lo referente a la caracterización de la asistencia psicológica en España, existen pocos estudios que describan la realidad de la psicología clínica. Uno de los principales contextos en los que se han llevado a cabo este tipo de investigaciones son las Clínicas Universitarias de Psicología. Desde que en 1984 y 1985 se crearan los primeros servicios de asistencia psicológica en la Universidad de Santiago de Compostela y en la Universidad de Barcelona (García-Vera, 2004; Saúl, LópezGonzález, \& Bermejo, 2009), han sido numero- sas las universidades que han desarrollado sus propios centros. Entre los objetivos de estos centros psicológicos universitarios destacan la asistencia psicológica, la formación y la investigación, como propone la Asociación Española de Servicios Psicológicos y Psicopedagógicos Universitarios (AESPPU, 2009), a la que están adscritos la mayoría de ellos.

Una revisión de Saúl et al. (2009) sobre los servicios de atención psicopedagógica y psicológica universitarios españoles, revela que en 2008 había un SPPU en 51 de las 74 universidades de España; 36 de ellas públicas y 15 privadas. Entre los principales servicios que ofrecen, hay 11 (16\%) centros que ofrecen principalmente apoyo psicopedagógico, 37 (54\%) asistencia psicológica y 22 (30\%) ambos servicios. Dentro de la asistencia psicológica, es más frecuente la intervención psicológica (realizada en el $60 \%$ de estos servicios) que el asesoramiento o consejo psicológico $y$, aunque algunos centros siguen como referencia un modelo cognitivo-conductual, existe una gran variabilidad en los modelos terapéuticos empleados en estas unidades. Los equipos y las relaciones laborales que conforman estos centros son muy variables en tamaño, incluyendo servicios con un solo profesional que da apoyo a diversos campus hasta equipos amplios con numerosos directores y colaboradores. En general, son pocos los contratados y la mayoría son psicólogos con beca de colaboración o en prácticas, aunque también colaboran profesores universitarios que supervisan o realizan labores asistenciales y personal administrativo (García-Vera, 2004). La mayoría de los SPPU dependen del Vicerrectorado y son gratuitos 
o disponen de tasas reducidas aunque, como destacan Saúl et al. (2009), existe un amplio desconocimiento de estos centros.

En este mismo estudio, se informa que la demanda más habitual en las clínicas universitarias son los problemas de ansiedad, estado de ánimo, desadaptación, déficit de autoestima, habilidades sociales, ansiedad ante los exámenes y problemas de relación interpersonal. Sin embargo, la demanda es notablemente menor respecto a otros problemas específicos (García-Vera, 2004), lo que no sorprende teniendo en cuenta que sólo algunos servicios incluyen de forma concreta la atención de dificultades sexuales, alimentarias, neuropsicológicas, así como programas de prevención y tratamiento orientados a la promoción de salud, desarrollo de competencias y orientados a la población infanto-juvenil. Ciertamente, los SPPU han atendido tradicionalmente a la comunidad universitaria (profesores, alumnos, personal de administración y servicios, familiares directos), aunque algunos centros han ido ampliado sus servicios a la población general (Saúl et al., 2009).

Las clínicas universitarias de atención psicológica juegan un papel fundamental para la profesionalización y capacitación de los psicólogos. La formación de estudiantes de grado, posgrado y psicólogos residentes posibilita una especialización en el área clínica y de salud que se distingue por las supervisiones, las horas de práctica y las sesiones clínicas en grupo. Esta modalidad facilita la adquisición de los requisitos mínimos establecidos para la formación de los psicólogos clínicos propuestos por la Federación Europea de Asociaciones de Psicólogos
(EFPA, 1990). Es más, facilitaría la formación al servir de puente entre los contenidos académicos anteriores y la práctica profesional privada, la cual resulta ser mayoritaria (el 63\% de los psicólogos clínicos trabajan en centros privados) (Santolaya, Berdullas, \& Fernández-Hermida, 2002). Es más, las Clínicas Universitarias, dada su naturaleza asistencial e investigadora, son un escenario idóneo para favorecer la tan necesaria unión entre la psicología aplicada y la investigación y para encontrar un equilibrio entre las carencias de las investigaciones controladas y de los estudios en el campo aplicado. Las características de estas clínicas propician el estudio de la eficacia de los tratamientos (Borkovec, 2004) en la medida en que las intervenciones psicológicas están más protocolizadas, se hace una medición sistemática de los cambios clínicos y hay una supervisión y formación continuada de los terapeutas.

En términos de la efectividad de las intervenciones en las clínicas universitarias, los datos actuales de los centros universitarios nacionales e internacionales, aunque escasos, apoyan resultados positivos sobre sus intervenciones, con buenos niveles de efectividad y una mejoría significativa de los clientes (Bados, Balaguer, \& Saldaña, 2007a; Hiltunen, Kocys, \& Perrin-Wallqvist, 2013; Öst Karlstedt, \& Widén, 2012). En este sentido, disponer de terapeutas con poca experiencia no es un impedimento para la eficacia de las intervenciones y los resultados de los tratamientos psicológicos con apoyo científico, puesto que resultan adecuados y comparables en términos de efectividad a otros estudios de investigación (Estupiñá \& Labrador, 2012). 
En esta línea, en el presente estudio se describen las principales características de los tratamientos que se llevan a cabo en la Clínica Universitaria del Centro de Psicología Aplicada de la Universidad Autónoma de Madrid (CPAUAM). Concretamente, se detallan (1) los datos sociodemográficos y clínicos de las personas que acudieron al centro, así como (2) algunas variables relacionadas con el éxito terapéutico y el tipo de tratamientos y procedimientos clínicos utilizados. Estos datos permitirían ampliar la información relativa a la demanda de los servicios universitarios de asistencia psicológica españoles y sugerir algunas reflexiones sobre la importancia de la calidad asistencial e investigación desde estos centros.

\section{Método}

\section{Participantes}

En este estudio se analizan los datos de 309 personas que recibieron tratamiento psicológico en la Clínica Universitaria del CPA-UAM entre 2012 y 2015 . Todos ellos eran adultos entre 18 y 77 años, con una media de edad de 28,97 (DT $=11,43)$. Los casos de menores de edad se han excluido de este análisis. La mayor parte de los consultantes eran mujeres $(64,7 \%)$ y la nacionalidad predominante en la muestra fue la española (90\%). El estado civil mayoritario era el de soltero $(55,2 \%)$ y el nivel de estudios finalizado más frecuente era Bachillerato/BUP (46,3\%), ya que la mayor parte de la muestra estaba formada por estudiantes universitarios en activo. En la Tabla 1 puede verse un resumen de los datos sociodemográficos de la muestra.

En el presente estudio se muestran los datos de los casos registrados y de los tratamien- tos realizados por 20 psicólogos distintos, con formación de Máster en Psicología General Sanitaria y con un rango de edad entre 24 y 30 años. En el centro se aplican tratamientos reconocidos como eficaces en el ámbito de la intervención cognitivo-conductual en todas sus variantes, tratando de ajustarse a la demanda planteada, teniendo en cuenta las competencias de la persona y sus circunstancias personales y sociales.

\section{La Clínica Universitaria del CPA-UAM}

La Clínica Universitaria del CPA-UAM está reconocida desde 2014 como Centro Sanitario por la Comunidad de Madrid y presta servicios tanto a la comunidad universitaria (personal, estudiantes y familiares), como a personas externas a este ámbito. En todos los casos los usuarios pagan por el tratamiento en la horquilla de tarifas aprobadas por el Consejo Social de la UAM. La universidad financia en parte los tratamientos al personal universitario, estudiantes y familiares directos. A priori no existen criterios de exclusión para la atención psicológica salvo en casos de demandas concretas para las que no se cuenta con psicólogos especializados. En estos casos los clientes son derivados a centros con los que existe un convenio de colaboración.

El Centro cuenta con un equipo estable de profesores de la Facultad de Psicología de la UAM que atiende algunos casos y supervisa al equipo clínico, formado por psicólogos que recientemente acabaron su formación como Máster en Psicología General Sanitaria. Cada año se incorporan al equipo en torno a seis psicólogos nuevos y después del primer año 
Tabla 1

Características sociodemográficas de los consultantes

\begin{tabular}{|c|c|}
\hline Variab & $(n=309)$ \\
\hline Edad, años (Media, SD) & $28,97(11,43)$ \\
\hline Sexo, mujeres \% (n) & $64,7(200)$ \\
\hline \multicolumn{2}{|l|}{ Nacionalidad \% (n) } \\
\hline España & $90,0(224)$ \\
\hline Países de habla hispana & $4,8(12)$ \\
\hline Otros & $5,2(13)$ \\
\hline \multicolumn{2}{|l|}{ Estado civil \% (n) } \\
\hline Soltero & $55,2(170)$ \\
\hline Casado & $16,9(52)$ \\
\hline Con pareja sin convivencia & $15,9(49)$ \\
\hline Convivencia en parea & $7,5(23)$ \\
\hline Divorciado & $3,2(10)$ \\
\hline Viudo & $1,3(4)$ \\
\hline \multicolumn{2}{|l|}{ Nivel de estudios \% (n) } \\
\hline Bachillerato/BUP & $46,3(143)$ \\
\hline Universitarios & $29,1(90)$ \\
\hline Posgrado/doctorado & $12,3(38)$ \\
\hline Formación Profesional & $5,5(17)$ \\
\hline Educación Secundaria & $4,2(13)$ \\
\hline Educación Primaria & $2,3(7)$ \\
\hline
\end{tabular}

se selecciona a dos de ellos para que continúen su residencia en el centro por un total de cuatro años. El equipo de psicólogos nuevos realiza el trabajo asistencial bajo la supervisión directa del equipo de profesores, expertos en Psicología Clínica, así como bajo la supervisión de los psicólogos de segundo año; todos los psicólogos supervisores tienen como objetivo ayudar al afrontamiento del caso. Por su parte, los psicólogos de segundo, tercer y cuarto año dedican una amplia proporción de su tiempo a la atención de pacientes, además de otras labores de formación de alumnos en prácticas e investigación y, por otra parte, tienen reuniones regulares dedicadas a la supervisión de los casos que tratan. La supervisión se realiza mediante el análisis de las historias clínicas y a través de la observación directa de algunas de las grabaciones, realizadas por medio del circuito cerrado de cámaras instalado en el centro.

En cuanto a los protocolos de intervención del CPA-UAM, siempre se comienza por la realización de una primera sesión de evaluación a todos los usuarios por parte de los expertos responsables del centro para decidir si se puede atender la demanda en el equipo y asignar el caso a uno de los psicólogos del centro. Una vez que los casos comienzan el tratamiento, todos los psicólogos desarrollan sus historias clínicas por sesiones asociadas a las fechas de las mismas, en tres apartados: información, análisis histórico y funcional de las conductas problema relevantes para el tratamiento, $y$ tareas intersesiones para el cliente y para el propio terapeuta. 


\section{Variables analizadas e instrumentos}

Se analizaron las siguientes variables de la muestra clínica tratada en el centro:

- Número total de sesiones de cada caso.

- Tipo de problema: no se realizaron diagnósticos (clasificación DSM-5, 2013) en sentido estricto, sino que se utilizaron categorías de problemas psicológicos de uso clínico común entre expertos. En cada caso, los terapeutas utilizaron los instrumentos de evaluación que consideraron oportunos hasta llegar a elaborar el análisis funcional de cada cliente, a partir del cual se diseñó la propuesta individualizada de tratamiento.

- Motivo para finalizar el tratamiento: se consideraron las categorías alta terapéutica, abandono, sólo evaluación y derivación a otro centro.

- Satisfacción del usuario con el tratamiento: los usuarios del centro completaron un cuestionario anónimo de nueve ítems creado para evaluar la satisfacción con la atención profesional y administrativa del centro. En este estudio se muestran los resultados de satisfacción general con el tratamiento, que se midieron en una escala de 0 a 10.

- Nivel de funcionamiento general: los terapeutas valoraron el estado de sus clientes al iniciar y terminar el tratamiento utilizando la Escala de Evaluación de la Actividad Global (EEAG), presente en el Eje V del DSM-IV-TR (APA, 2000). Esta escala incluye la opinión del clínico acerca del nivel general de actividad del cliente teniendo en cuenta la actividad psicosocial, social y laboral. Permite valorar la gravedad del caso así como su evolución. Para ello el psicólogo debe utilizar un único valor que refleje del mejor modo posible el nivel global de actividad del individuo, entre 1 (peligro o incapacidad persistente) y 100 (actividad satisfactoria. Sin síntomas).

- Valoración de la eficacia del tratamiento según el terapeuta: los terapeutas valoraron de 0 (ineficaz) a 10 (totalmente eficaz) el logro de los objetivos establecidos en el tratamiento según su criterio profesional consensuado con el equipo del CPA-UAM.

\section{Procedimientos de registro y análisis de los casos atendidos}

Los datos relativos a los tratamientos llevados a cabo en el CPA-UAM fueron introducidos por cada uno de los terapeutas que llevaron a cabo el tratamiento en la base de datos. Esta base de datos cuenta con instrucciones de cumplimentación detalladas para cada variable y todos los psicólogos del centro reciben al entrar formación para poder completar la información.

Todos los clientes fueron informados de que sus datos podían ser utilizados con fines docentes y de investigación y dieron su consentimiento por escrito en estricto cumplimiento de la Ley de Protección de Datos.

Para los análisis que aquí se presentarán, se realizó una depuración de la base de datos del centro excluyéndose los registros relativos a trastornos de la conducta alimentaria y casos infantiles. Para el análisis de la tipología y variables sociodemográficas se utilizó toda la muestra registrada $(\mathrm{N}=309)$ y para el análisis del tratamiento y su eficacia se analizaron 106 de estos casos, que corresponden a aquellos cuyo tratamiento estaba finalizado y cuyos registros 
estaban completos en todos sus campos. Una vez completada la depuración se realizaron análisis descriptivos de todas las variables incluidas en el estudio, así como comparación de medias entre las puntuaciones iniciales y finales en la escala EEAG y se establecieron correlaciones entre algunas variables de interés clínico.

\section{Resultados}

En primer lugar, se presentan los datos de los casos atendidos en la Clínica del CPA-UAM en el periodo estudiado ( $N=309)$ y sus características sociodemográficas por tipología clínica. Posteriormente, se presentarán los datos de eficacia del tratamiento de los 106 casos registrados en todos los campos y cuyos tratamientos estaban finalizados.
Los problemas clínicos más frecuentes que se atienden en el CPA son de ansiedad (36,8\%), seguidos por los de bajo estado de ánimo $(13,3 \%)$ y ambos combinados (8,3\%). Como problemas menos frecuentes, encontramos las adicciones a sustancias (0,3\%), los trastornos de personalidad $(0,6 \%)$ y los trastornos psicóticos (0,3\%). En la Tabla 2 se detalla la frecuencia con que se presenta cada tipo de problema en la muestra, así como algunos datos sociodemográficos y relativos a la eficacia de los tratamientos para cada tipología. Tal como se puede apreciar, entre los problemas más frecuentes, la tasa de alta terapéutica siempre es muy superior a la tasa de abandono.

En el momento de realizar el estudio el $82,5 \%(n=255)$ de los pacientes ya no estaba

Tabla 2

Tipología de problemas tratados y eficacia de los tratamientos

\begin{tabular}{|c|c|c|c|c|c|}
\hline Tipo de problama psicológico & $\begin{array}{l}\text { Casos } \\
\text { registrados } \\
\%(n)\end{array}$ & $\begin{array}{l}\text { Edad } \\
\text { M (SD) }\end{array}$ & $\begin{array}{l}\text { Porcentaje de } \\
\text { mujeres }\end{array}$ & $\begin{array}{l}\text { Porcentaje de } \\
\text { altas }\end{array}$ & $\begin{array}{l}\text { Porcentaje de } \\
\text { abandonos }\end{array}$ \\
\hline Problemas de ansiedad & $36,8(116)$ & $28,46(11,24)$ & 74,1 & 60,9 & 17,2 \\
\hline Bajo estado de ánimo & $13,3(42)$ & $30,31(11,79)$ & 66,7 & 50 & 28,6 \\
\hline Ansiedad y bajo estado de ánimo & $8,3(26)$ & $31,85(12,77)$ & 73,1 & 73,1 & 7,7 \\
\hline Habilidades y relaciones sociales & $7,6(24)$ & $28,33(12,26)$ & 58,3 & 58,3 & 29,2 \\
\hline Problemas de pareja & $5,7(18)$ & $36,82(15,61)$ & 76,5 & 44,4 & 33,3 \\
\hline Otros & $5,1(16)$ & $26,80(10,51)$ & 37,5 & 68,8 & 12,5 \\
\hline $\begin{array}{l}\text { Problemas relacionados con el } \\
\text { trabajo y el estudio }\end{array}$ & $4,8(15)$ & $23,53(7,35)$ & 46,7 & 33,3 & 53,3 \\
\hline Problemas sexuales & $3,8(12)$ & $25,86(6,71)$ & 58,3 & 41,7 & 33,3 \\
\hline Relaciones paterno-filiares & $2,5(8)$ & $23,38(5,80)$ & 75,0 & 50 & 25 \\
\hline Ira / Agresividad & $2,2(7)$ & $32,71(13,12)$ & 42,9 & 14,3 & 28,6 \\
\hline Bipolar & $1,6(5)$ & $32,40(13,43)$ & 40,0 & 20,0 & 60,0 \\
\hline Problemas de sueño & $1(3)$ & $24,33(8,39)$ & 33,3 & 66,6 & 33,3 \\
\hline Adicciones comportamentales & $1(3)$ & $31,00(17,32)$ & 66,7 & 0 & 66,7 \\
\hline Trastornos de personalidad & $0,6(2)$ & $21,50(0,71)$ & 33,3 & 50 & 50 \\
\hline Adicciones a sustancias & $0,3(1)$ & 30 & 0 & 100 & 0 \\
\hline Psicóticos & $0,3(1)$ & 25,00 & 0 & 0 & 100 \\
\hline
\end{tabular}


en tratamiento; de los cuales, el 65,1\% (166) había obtenido alta terapéutica y el 29,8\% (76) había abandonado el tratamiento. Un 3,1\% había sido derivado a otros centros y un $2 \%$ únicamente había consultado para evaluación. El $16,5 \%$ restante todavía estaba en alguna de las fases de la intervención $(n=51)$.

En los casos que acabaron el tratamiento en el CPA con alta $(n=166)$, encontramos que el número total de sesiones osciló entre 3 y 60 , siendo la media de sesiones 17,01 ( $S D=8,09$ ). Atendiendo a las diferentes fases de la intervención, el número de sesiones de evaluación osciló entre 1 y 8 , siendo lo más frecuente 3 sesiones $(M=3,32, S D=1,20)$; el número medio de sesiones de tratamiento fue de 12,09 (Rango $=1-50 ; S D=7,40) ; y$, por último, las sesiones de seguimiento variaron entre 0 y 9 , con una media de 1,62 sesiones $(S D=1,51$ ).

La eficacia del tratamiento fue también evaluada mediante la escala de valoración EEAG, la satisfacción del usuario con el tratamiento y la valoración del éxito del tratamiento según el terapeuta. La muestra con todos los datos completos es más reducida $(\mathrm{N}=106)$. Se encontró que comparando el EEAG inicial con el EEAG al finalizar el tratamiento existe una mejora media de 28,26 puntos ( $S D=13,23$ ) que resulta significativa $(t(148)=26,07, p<0,001)$. El EEAG medio inicial es de $61,18(S D=11,67)$, que corresponde a la siguiente definición "Síntomas moderados (p. ej., afecto aplanado y lenguaje circunstancial, crisis de angustia ocasionales) o dificultades moderadas en la actividad social, laboral o escolar (p. ej., pocos amigos, conflictos con compañeros de trabajo o de escuela)"; y el final es de $89,44(S D=8,19)$ que corresponde a la siguiente descripción "Actividad satisfactoria en una amplia gama de actividades, nunca parece superado por los problemas de su vida, es valorado por los demás a causa de sus abundantes cualidades positivas. Sin síntomas".

En cuanto a la satisfacción del usuario con el tratamiento, la valoración media del éxito del tratamiento según el consultante es de 9,49 puntos $(S D=0,83)$ en una escala de 0 a 10. Y, en cuanto la valoración del éxito del tratamiento según el terapeuta, la valoración media por parte de los terapeutas es 9,02 puntos $(\mathrm{SD}=1,02)$.

\section{Discusión}

El perfil sociodemográfico de los clientes que acuden al CPA-UAM es similar al observado por otros servicios estudiados (Bados, Balaguer, \& Saldaña, 2007a y b; Hiltunen, Kocys, \& Perrin-Wallqvist, 2013; Labrador, Estupiñá, \& Vera 2010; Labrador et al., 2016; Valero \& Ruiz, 2003). La existencia de un perfil más joven, de mujer soltera y con un nivel de estudios más alto, en comparación con otro tipo de servicios de psicología, probablemente se deba al contexto universitario en el que se ubica la clínica.

Atendiendo a las demandas más habituales, destaca que los problemas de ansiedad y estado de ánimo suponen más de la mitad de los motivos de asistencia psicológica, como se observa en otros centros similares (Labrador et al., 2016; Saúl, 2009). Los resultados constatan la presencia de otras problemáticas también habituales en centros universitarios, tales como problemas de de habilidades y relaciones sociales, problemas relacionados con el estudio o el trabajo o dificultades de pareja, 
entre otros. Además, se confirma la escasa representación que tienen otras problemáticas específicas, como por ejemplo, los problemas psicóticos (García-Vera, 2004). Es destacable la alta presencia de dificultades de estudio o laborales en nuestra muestra en comparación con otras muestras, que probablemente queden tan representados por la importante presencia de estudiantes y trabajadores procedentes de la universidad, pese a que el centro atiende también a la población general. No obstante, los datos no reflejan una distribución epidemiológica similar de la población general aportada por otros estudios: problemas depresivos, seguidos de fobias específicas, abuso de alcohol y distimia (Haro et al., 2006). Analizando las diferencias en la distribución de la muestra en función de las distintas problemáticas, se puede destacar que en la mayoría de los problemas menos frecuentes (trastorno bipolar, trastorno psicótico, problemas de sueño, trastornos de personalidad y adicciones) la muestra está compuesta por más hombres que mujeres, a diferencia de lo que ocurre en los problemas más frecuentes de nuestra muestra. Sin embargo, al ser el número de estos problemas tratados tan pequeño en el CPA-UAM, conviene ser cautos al tomar en consideración estos datos.

Respecto a la duración de los tratamientos psicológicos, en nuestra clínica la media de sesiones de tratamiento se sitúa en torno a 17, que incluye una media de sesiones de evaluación e intervención de tres y 12 sesiones, respectivamente. Esta duración es similar a la duración en otras clínicas estudiadas, con valores que se mueven en una horquilla desde las 11 sesiones (Hiltunen, Kocys, \& Perrin-Wallqvist, 2013), hasta las 18 sesiones o menos (Labrador et al., 2016) o incluso las 27 sesiones (Bados et al., 2007a). A pesar de la corta experiencia de los terapeutas, no se da una mayor duración de la intervención aplicada, como cabría esperar (Bados et al., 2007a). Se observa una alta variabilidad en el número de sesiones por fase de tratamiento y totales, que con alta probabilidad se deba a los diferentes tipos de demandas y las áreas afectadas en cada caso (individual, laboral, social, familiar, etc.). Destaca que una parte importante del tratamiento psicológico, siendo éste breve, se dedica principalmente a la evaluación. Se entiende que esto podría estar relacionado con que un mayor esfuerzo y tiempo de evaluación, redundaría en un tratamiento más breve y eficaz. Aunque, como señalan algunos autores, esto pudiera restar competitividad y resultar menos motivador para los clientes (Labrador et al., 2016). En general, es posible que el uso de tratamientos empíricamente validados contribuya a la brevedad que presentan los mismos (Hiltunen, Kocys, \& Perrin-Wallqvist, 2013; Labrador et al., 2016).

En cuanto a la eficacia de las intervenciones terapéuticas, aunque no existe acuerdo unánime sobre las variables que deben analizarse, los índices de altas terapéuticas y abandonos son el elemento que generalmente se considera. Los datos reflejan que una alta proporción de las personas que iniciaron una terapia obtuvieron alta terapéutica, mientras que un $29,8 \%$ abandonaron la intervención. Estos porcentajes son similares a los hallados en otros contextos clínicos universitarios, como el 
31,7\% de abandono encontrado en la Clínica de la Universidad Complutense de Madrid (Labrador et al., 2016), e inferiores al 43,8\% de la Unidad de Terapia de Conducta de la Universidad de Barcelona (Bados, Balaguer, \& Saldaña, 2007b). Swift y Greenberg (2012) obtuvieron en su metaanálisis una tasa media de abandono del 19,7\%, aunque lo más llamativo fue la gran heterogeneidad de dichas tasas, entre $0 \%$ y $74,23 \%$. El abandono terapéutico es un problema común en la práctica clínica, no solamente en los contextos universitarios, y se da con unas tasas similares. Algunos autores señalan que las variables relacionadas con el abandono serían razones económicas, insatisfacción con el tratamiento recibido, escasa motivación, larga duración del tratamiento, $y$, en general, la falta de coincidencia entre lo que el paciente espera y lo que se le ofrece (Bados, García, \& Fusté, 2002); otros han señalado como variables moduladoras el diagnóstico, la edad del paciente y el nivel de experiencia del terapeuta (Swift \& Greenberg, 2012). En nuestro caso, las características de la población con la que se ha trabajado, esencialmente vinculada al contexto universitario de forma transitoria, pueden estar influyendo también en el abandono del tratamiento, pues la finalización del periodo académico obliga, en muchos casos, a finalizar la terapia de forma prematura.

Para valorar la eficacia de los tratamientos también se utilizó, en este caso, las puntuaciones del EEAG previo y posterior a la terapia. Se encontró una mejora notable, que va asociada a una mejoría en la actividad psicológica, social y ocupacional de los usuarios. Consideramos que esta escala es una medida simple y prácti- ca para valorar aspectos relevantes y globales del individuo que complementa el análisis de la eficacia de las intervenciones psicológicas. Por otra parte, se encontró que la satisfacción de los usuarios con el tratamiento recibido fue muy elevada, así como la valoración del éxito del tratamiento según el terapeuta, ambos aspectos subjetivos de gran relevancia clínica.

En numerosos trabajos científicos se han señalado los factores individuales del terapeuta como algunos de los que, invariablemente, afectan al resultado de los tratamientos psicológicos (Norcross \& Lambert, 2011). La experiencia de los psicólogos es una de estas variables señaladas en numerosos estudios, entre otras muchas. No se puede negar la influencia de la experiencia, pero datos como los que se presentan en este estudio ponen de manifiesto que incluso terapeutas con poca experiencia, si son supervisados adecuadamente por otros de mayor experiencia, podrían conseguir buenos resultados en los tratamientos aplicados.

Un aspecto que también es importante valorar en las intervenciones psicológicas es la eficiencia de las mismas. Para abordar este aspecto, hemos realizado un cálculo del coste medio de la terapia en el CPA-UAM: teniendo en cuenta el número medio de sesiones (17 sesiones), ésta tendría un coste de entre 476 $€$ y $850 €$ en función de la relación que tenga el usuario con la universidad. Los costes de la intervención en términos temporales tendrían una duración aproximada de 6 meses, teniendo en cuenta los períodos de seguimiento del proceso terapéutico. Comparando nuestro centro universitario con el de la Universidad Complutense de Madrid (Labrador et al., 
2016), que hace el análisis de sus tratamientos en estos mismos términos, se encuentran datos similares (826 euros). En comparación, los precios aplicados en clínicas privadas suelen ser más altos, más variables $y$, además, poco accesibles.

Si se atiende al Sistema Público de Salud, los datos evidencian que los trastornos psicológicos tienen un enorme impacto socioeconómico a nivel nacional y europeo. Por ejemplo, los trastornos del estado de ánimo suponen el mayor coste económico a nivel europeo, representando un coste anual de 113.405 miIlones de euros (Olesen, Gustavsson, Svensson, Wittchen, \& Jönsson, 2012; Parés-Badell et al., 2014). Tanto a nivel nacional como internacional existe evidencia suficiente que revela que la terapia psicológica es un recurso eficaz y eficiente tanto para población adulta como infanto-juvenil, pues consigue reducir el número de visitas al médico y el gasto sanitario, reduce la discapacidad, la morbilidad y la mortalidad, mejora el funcionamiento en el trabajo, disminuye los días de hospitalización psiquiátrica, es una intervención más rentable a largo plazo y tiene un menor coste que el tratamiento farmacológico (APA, 2013; Echeburúa, Corral, \& Salaberría, 2010). Teniendo en cuenta este escenario, consideramos interesante disponer de una atención psicológica rentable y eficiente a través de las clínicas universitarias por el bajo coste que supone y los beneficios psicológicos que aporta.

En lo que respecta a las limitaciones del presente estudio, así como a las posibles mejoras que podrían realizarse, existen varios aspectos a destacar. El tamaño de la muestra es pequeño y existe un elevado número de datos perdidos que sería deseable mejorar en futuros estudios; estos datos perdidos se pueden explicar por el elevado coste de tiempo que supone para los terapeutas llevar al día una base de datos extensa sobre sus casos. La escala EEAG que se presenta, aunque puede ser una primera aproximación a la valoración de la eficacia de los tratamientos, es insuficiente y en futuros estudios nos gustaría contar con puntuaciones pre-post tratamiento mediante cuestionarios estandarizados. Por otra parte, a pesar de tener una tasa de abandono semejante a la encontrada en otros entornos clínicos, consideramos que debemos primar el objetivo de reducirla, estudiando con detalle los motivos de abandono e identificando aquellos aspectos sobre los que se puede intervenir. Por último, en la clínica del CPA-UAM no se categorizan los problemas atendiendo a los criterios diagnósticos del DSM-5, lo cual supone una limitación a nivel de investigación, ya que puede limitar las comparaciones de datos con otros estudios. Sin embargo, esta decisión nos ha permitido recoger para este estudio todos los problemas de los pacientes atendiendo a la descripción que realiza el clínico del tipo de problema, no dejando fuera problemas subclínicos; estos datos han llegado a suponer el 15,4\% en alguna muestra (Labrador et. al, 2016). La decisión de no utilizar criterios DSM tiene que ver con que el CPAUAM considera el uso del análisis funcional de la conducta un pilar básico en la formación clínica de los psicólogos residentes, para garantizar la calidad y eficacia de sus tratamientos.

Entendemos que los datos de este primer artículo del CPA-UAM aportan un conocimien- 
to de inestimable valor al mostrar el trabajo realizado con intervenciones estandarizadas y eficaces, con tratamientos de elevada rentabilidad temporal y económica. Se perfilan así las Clínicas Universitarias como un recurso competitivo frente a otro tipo de tratamientos en salud mental (farmacológicos o alternativos), un recurso sobresaliente para la mejora del bienestar psicológico de la población.

\section{Referencias}

American Psychiatric Association (1992). Ethical principles of psychologist and code of conduct. Recuperado de http://www.apa. org/ethics/code/

American Psychiatric Association (2000). Diagnostic and statistical manual of mental disorders: DSM-IV-TR (4a ed., texto rev.). Washington D. C.: Author. doi:10.1176/appi. books.9780890423349

American Psychiatric Association (2013). Diagnostic and statistical manual of mental disorders: DSM-5 (5a ed.). Washington D. C.: Author. doi:10.1007/springerreference_179660

Bados, A., Balaguer, G., \& Saldaña, C. (2007a). Outcome of cognitive-behavioural therapy in training practice with anxiety disorder patients.British JournalofClinical Psychology, 46, 429-435. doi:10.1348/014466507X209961

Bados, A., Balaguer, G., \& Saldaña, C. (2007b). The efficacy of cognitive-behavioral therapy and the problem of drop-out. Journal of Clinical Psychology, 63, 585-592. doi:10.1002/ jclp.20368

Bados, A., García, E., \& Fusté, A. (2002). Eficacia y utilidad clínica de la terapia psicológica. International Journal of Clinical and Health Psychology, 2, 477-502.

Becoña, E. (1999). La discrepancia entre la investigación y la práctica clínica de la terapia de conducta. Revista de Psicopatología y Psicología Clínica, 4, 71-103. doi:10.5944/rppc. vol.4.num.2.1999.3875

Bones, K., Pérez, K., Rodríguez-Sanz, M., Borrell, C., \& Obiols, J. (2010). Prevalencia de proble- mas de salud mental y su asociación con variables socioeconómicas, de trabajo y salud: resultados de la Encuesta Nacional de Salud de España. Psicothema, 22, 389-395.

Borkovec, T. D. (2004). Research in training clinics and practice research networks: A route to the integration of science and practice. Clinical Psychology: Science and Practice, 11, 211-215. doi:10.1093/clipsy/bph073

Bower, P. (2003). Efficacy in evidence based practice. Clinical Psychology \& Psychotherapy, 10, 328-336. doi:10.1002/cpp.380

Chambless, D., \& Ollendick, T. (2001). Empirically supported psychological interventions: ÇControversies and evidence. Annual Review of Psychology, 52, 685- 716. doi:10.1146/annurev.psych.52.1.685

COP (1993). Código deontológico del psicólogo. Recuperado de http://www.copmadrid. org/web/files/ciudadanos_codigodeontologico.pdf

Echeburúa, E., Corral, P., \& Salaberría, K. (2010). Efectividad de las terapias psicológicas: Un análisis de la realidad actual. Revista de Psicopatología y Psicología Clínica, 15, 85-99. doi:10.5944/rppc.vol.15.num.2.2010.4088

Asociación Española de Servicios Psicológicos y Psicopedagógicos Universitarios (AESPPU, 2009). Estatutos. Recuperado de http://ice2. uab.cat/UAP/aesppu/docs/estatutos.pdf

Estupiñá, F. J., \& Labrador, F. J. (2012). Effectiveness of cognitive-behavioral treatment for major depressive disorder in a university psychology clinic. The Spanish Journal of Psychology, 15, 1388-1399. doi:10.5209/ rev_sjop.2012.v15.n3.39423

European Federation of Psychologists' Associations (EFPA, 1990). Optimal standards for profesional training in Psychology. Recuperado de http://www.efpa.eu/professionaldevelopment/optimal-standards-for-professional-training-in-psychology

García Vera, M. P. (2004). Los centros universitarios de asistencia psicológica como centros de formación de posgrado en psicología clínica y psicoterapia: la Unidad de Psicología Clínica y de la Salud de la UCM. Papeles del Psicólogo, 87, 59-66.

Haro, J. M., Palacín, C., Vilagut, G., Martínez, 
M., Bernal, M., Luque, I., \& Alonso, J. (2006). Prevalencia de los trastornos mentales y factores asociados: resultados del estudio ESEMeD-España. Medicina Clínica, 126, 445451. doi:10.1157/13086324

Hiltunen, A. J., Kocys, E., \& Perrin-Wallqvist, R. (2013). Effectiveness of cognitive behavioral therapy: An evaluation of therapies provided by trainees at a university psychotherapy training center. PsyCh Journal, 2, 101112. doi:10.1002/pchj.23

Kazdin, A. E. (2008). Evidence-based treatment and practice: New opportunities to bridge clinical research and practice, enhance the knowledge base, and improve patient care. American Psychologist, 63, 146. doi:10.1037/0003-066x.63.3.146

Labrador, F. J., Bernaldo-de-Quirós, M., GarcíaFernández, G., Estupiñá, F., Fernández-Arias, I., \& Labrador-Méndez, M. (2016). Characteristics of demand and psychological treatments in a university clinic. Clínica y Salud, 27, 7-14. doi:10.1016/j.clysa.2016.01.002 Labrador, F. J., Fernández, P., \& Ballesteros, F. (2011). Tratamientos psicológicos en la práctica clínica cotidiana. Anales de Psicología, 27, 319-326.

Labrador, F. J., Estupiñá, F. J., \& Vera, M. P. G. (2010). Demanda de atención psicológica en la práctica clínica: tratamientos y resultados. Psicothema, 22, 619-626.

Norcross, J. C., \& Lambert, M. J. (2011). Evidence-based therapy relationships. En J. C. Norcross (Ed.), Psychotherapy relationships that work. Evidence-based responsiveness ( $2^{\mathrm{a}}$ ed.) (pp. 3-21). Nueva York: Oxford University Press.

Olesen, J., Gustavsson, A., Svensson, M., Wittchen, H. U., \& Jönsson, B. (2012). The economic cost of brain disorders in Europe. European Journal of Neurology, 19, 155-162. doi:10.1111/j.1468-1331.2011.03590.x

Öst, L. G., Karlstedt, A., \& Widén, S. (2012). The effects of cognitive behavior therapy delivered by students in a psychologist training program: An effectiveness study. Behavior Therapy, 43, 160-173. doi:10.1016/j. beth.2011.05.001
Parés-Badell O., Barbaglia G., Jerinic P., Gustavsson A., Salvador-Carulla L., \& Alonso, J. (2014). Cost of disorders of the brain in Spain. PLoS ONE 9, e105471. doi:10.1371/ journal.pone.0105471

Pérez Álvarez, M., Fernández Hermida, J. R., Fernández Rodríguez, C., \& Amigo Vázquez, I. (2003). Guía de tratamientos psicológicos eficaces I. Adultos. Madrid: Pirámide.

Santolaya, F., Berdullas, M., \& Fernández-Hermida, J. R. (2002). La década 1989-1998 en la Psicología Española: análisis del desarrollo de la psicología profesional en España. Papeles del Psicólogo, 82, 65-82.

Saúl Gutiérrez, L. Á., López-González, M. D. L. A., \& Bermejo, B. G. (2009). Revisión de los servicios de atención psicológica y psicopedagógica en las universidades españolas. Acción psicológica, 6, 17-40. doi:10.5944/ ap.6.1.227

Shean, G. D. (2012). Some limitations on the external validity of psychotherapy efficacy studies and suggestions for future research. American Journal of Psychotherapy, 66, 227242.

Swift, J. K., \& Greenberg, R. P. (2012). Premature discontinuation in adult psychotherapy: A meta-analysis. Journal of consulting and clinical psychology, 80, 547. doi:10.1037/ a0028226

Valero, L., \& Ruiz, M. A. (2003). Evaluación de un servicio de salud mental: análisis de la demanda y datos epidemiológicos. Psiquis, 24, 11-1.

Westen, D. (2007). Discovering what works in the community: Toward a genuine partnership of clinicians and researchers. En S. G. Hofmann, \& J. Weinberger (Eds.), The art and science of psychotherapy (pp. 3-29). New York, NY: Routledge, Taylor \& Francis Group. 